\title{
The HST large programme on $\omega$ Centauri - I. Multiple stellar populations at the bottom of the main sequence probed in NIR-Optical
}

\author{
A. P. Milone, ${ }^{1 \star}$ A. F. Marino, ${ }^{1}$ L. R. Bedin, ${ }^{2}$ J. Anderson, ${ }^{3}$ D. Apai, ${ }^{4,5}$ A. Bellini, ${ }^{3}$ \\ P. Bergeron, ${ }^{6}$ A. J. Burgasser, ${ }^{7}$ A. Dotter ${ }^{8}$ and J. M. Rees ${ }^{4}$ \\ ${ }^{1}$ Research School of Astronomy and Astrophysics, Australian National University, Mt Stromlo Observatory, via Cotter Rd, Weston, ACT 2611, Australia \\ ${ }^{2}$ Istituto Nazionale di Astrofisica - Osservatorio Astronomico di Padova, Vicolo dell'Osservatorio 5, IT-35122 Padova, Italy \\ ${ }^{3}$ Space Telescope Science Institute, 3800 San Martin Drive, Baltimore, MD 21218, USA \\ ${ }^{4}$ Department of Astronomy and Steward Observatory, The University of Arizona, 933 N. Cherry Avenue, Tucson, AZ 85721, USA \\ ${ }^{5}$ Lunar and Planetary Laboratory, The University of Arizona, 1640 E. University Blvd., Tucson, AZ 85721, USA \\ ${ }^{6}$ Département de Physique, Université de Montréal, C.P. 6128, Succ. Centre-Ville, Montréal, QC H3C 3J7, Canada \\ ${ }^{7}$ Center for Astrophysics and Space Science, University of California, San Diego, La Jolla, CA 92093, USA \\ ${ }^{8}$ Harvard-Smithsonian Center for Astrophysics, Cambridge, MA 02138, USA
}

Accepted 2017 March 30. Received 2017 March 30; in original form 2017 January 19

\begin{abstract}
As part of a large investigation with Hubble Space Telescope to study the faintest stars within the globular cluster $\omega$ Centauri, in this work we present early results on the multiplicity of its main sequence (MS) stars, based on deep optical and near-infrared observations. By using appropriate colour-magnitude diagrams, we have identified, for the first time, the two main stellar Populations I and II along the entire MS, from the turn-off towards the hydrogenburning limit. We have compared the observations with suitable synthetic spectra of MS stars and conclude that the two main sequences (MSs) are consistent with stellar populations with different metallicity, helium and light-element abundance. Specifically, MS-I corresponds to a metal-poor stellar population $([\mathrm{Fe} / \mathrm{H}] \sim-1.7)$ with $Y \sim 0.25$ and $[\mathrm{O} / \mathrm{Fe}] \sim 0.30$. The MS-II hosts helium-rich $(Y \sim 0.37-0.40)$ stars with metallicity ranging from $[\mathrm{Fe} / \mathrm{H}] \sim-1.7$ to -1.4 . Below the MS knee $\left(m_{F 160 W} \sim 19.5\right)$, our photometry reveals that each of the two main MSs hosts stellar subpopulations with different oxygen abundances, with very O-poor stars ([O/Fe] $\sim-0.5$ ) populating the MS-II. Such a complexity has never been observed in previous studies of M-dwarfs in globular clusters. A few months before the launch of the James Webb Space Telescope, these results demonstrate the power of optical and near-infrared photometry in the study of multiple stellar populations in globular clusters.
\end{abstract}

Key words: Hertzsprung-Russell and colour-magnitude diagrams - stars: low-mass - stars: Population II-globular clusters: general-globular clusters: individual: $\omega$ Centauri, M4, NGC2808.

\section{INTRODUCTION}

The most massive globular cluster (GC) of the Milky Way, $\omega$ Centauri, hosts a very complex system of multiple stellar populations that makes it one of the most enigmatic stellar systems of the Galaxy. In contrast with the majority of monometallic GCs, stars in $\omega$ Centauri span a wide range in metallicity, including populations with $[\mathrm{Fe} / \mathrm{H}]>-0.7$ (e.g. Norris \& Da Costa 1995; Pancino et al. 2002; Johnson et al. 2008, 2009; Marino et al. 2010, 2011).

^E-mail: milone@mso.anu.edu.au
It exhibits extreme star-to-star variation in several light elements, including $\mathrm{C}, \mathrm{N}, \mathrm{O}, \mathrm{Na}, \mathrm{Mg}, \mathrm{Al}$ and $\mathrm{Si}$ and s-process elements (e.g. Brown \& Wallerstein 1993; Norris \& Da Costa 1995; Johnson \& Pilachowski 2010; Stanford, Da Costa \& Norris 2010; Marino et al. 2012), and shows the typical abundance patterns among light elements that are observed in nearly all the Galactic GCs, such as the $\mathrm{Na}-\mathrm{O}$ and $\mathrm{C}-\mathrm{N}$ anticorrelations. Interestingly, in this extreme cluster distinct anticorrelations are present within stellar populations with different metallicity (Johnson \& Pilachowski 2010; Marino et al. 2010, 2011).

One of the most intriguing discoveries of the last decade in the field of stellar astrophysics has been that the MS of $\omega$ Centauri is split into a blue and a red component (Anderson 1997; Bedin 
et al. 2004) with the blue MS being more metal-rich than the red MS (Piotto et al. 2005). These facts have demonstrated that this cluster exhibits a large variation in helium, with the blue MS being highly enhanced in helium up to $Y \sim 0.39$ (e.g. Norris 2004; King et al. 2012). More recent papers have shown that the MS is even more complex than we had previously imagined and that both the blue and the red MSs host stellar subpopulations (e.g. Bellini et al. 2010). The complexity of multiple stellar populations in this cluster is further demonstrated by the multiple sub-giant branches (SGBs) and red-giant branches (RGBs) and by the presence of a double white dwarf (WD) cooling sequence (Lee et al. 1999; Pancino et al. 2000; Sollima et al. 2005; Villanova et al. 2007; Bellini et al. 2010; 2013; Milone et al. 2017).

In this paper, we use data covering one of the three parallel fields from one of the two epochs monitored by the Hubble Space Telescope (HST) under large programme GO-14118+14662 (PI: Bedin). A detailed description of the project and of the entire data set is provided in the paper by Bedin et al. (in preparation). For this field, deep optical data exists in the archive (see Section 2 for details) and it is possible to determine cluster membership. We exploit visual and near-infrared (NIR) $H S T$-photometry to investigate multiple stellar populations along the entire MS of $\omega$ Centauri, from the turn-off approaching the H-burning limit.

The paper is organized as follows: in Section 2, we describe the data and the data reduction. The colour-magnitude diagram (CMD) and its multiple sequences are presented in Sections 3 and 4, while in Section 5 we compare the observed colours of the distinct stellar populations with those predicted from appropriate synthetic spectra. A summary and a discussion of the main results are provided in Section 6.

\section{DATA AND DATA ANALYSIS}

The HST large programme GO-14118+14662 aims at observing the entire WD cooling sequence of $\omega$ Centauri. It uses the Wide Field Channel (WFC) of the Advanced Camera for Surveys (ACS) to investigate how the unusually large helium content of some of the stars impacts the evolution of those stars as WDs.

The field decontamination from background and foreground objects is particularly important when studying faint stars at sparse evolutionary phases in star clusters. Therefore, the programme was designed to collect observations at two epochs. While the first epoch from GO-14118 has just concluded, the second epoch from GO14662 will be collected between late 2017 and mid-2018.

In each of these two epochs, the 66 orbits staring at the main field are collected at three different orientations, with the goal of minimizing the impact of imperfect Charge-Transfer Efficiency, imperfect calibrations and artefacts, to have a better handle on systematic errors in astrometry and photometry.

As ancillary science, parallel fields with both the NIR and the UV-Visual (UVIS) channels of the Wide Field Camera 3 (WFC3) were also approved for a number of scientific subprogrammes. One of these scientific goals is the study of the multiple MS in the vicinity of the hydrogen-burning limit.

For each of the three orientations, the staring lasts 22 orbits. These are observed with both WFC3/UVIS (eight orbits) and WFC3/NIR (14 orbits). Although these fields are not deep enough to reach the end of the WD cooling sequence, they are deep enough to study the faintest regions of the MSs of $\omega$ Centauri, particularly when using WFC3/NIR, where cool stars are relatively brighter.

\subsection{The south-west parallel field at 17 arcmin}

During phase II, one of the three above-mentioned parallel fields was accurately placed to match relatively deep (GO-14118, four orbits) archival observations collected in 2002 and 2005 with ACS/WFC (more details will be presented in the following sections). For completeness, this field was not chosen as the main ACS/WFC field due to its relatively low density of cluster stars. This field is centred at $(\alpha ; \delta) \simeq(13: 25: 36.8 ;-47: 40: 05)$, that is about 17 arcmin from the cluster centre. This field is the focus of this paper.

In this work, we use only the WFC3/NIR images from GO-14118 collected between 2015 August 19 and August 26, and consisting of seven short exposures of $142 \mathrm{~s}$ and 14 long exposures of $1302 \mathrm{~s}$ for each of the two filters $F 110 \mathrm{~W}$ and $F 160 \mathrm{~W}$. The adopted exposure times have been chosen to maximize the dynamical range and optimize the detection of faint sources. ${ }^{1}$

Data were reduced with an adaptation to WFC3/NIR images of the software presented and described in Anderson et al. (2008) for the case of the ACS/WFC camera. The software is essentially a Point-Spread Function-fitting algorithm that performs simultaneous fitting of all the sources in a given patch of sky, by simultaneously using all the available images for that patch of sky, once transformed into a common reference frame. To achieve this, the software makes use of the publicly available PSFs and geometric-distortion solution for WFC3/NIR provided by Jay Anderson. ${ }^{2}$

\subsection{Archival material}

The parallel field considered in this work was previously observed with ACS/WFC on 2002 July 7 under programme GO-9444, and this is the field in which the MS split of $\omega$ Centauri was first demonstrated (Bedin et al. 2004). The data consist of two orbits per filter, $F 606 W$ and $F 814 W$, for a total of four $\sim 1300$ s deep exposures per filter. A second epoch was awarded to obtain high-precision propermotion membership (GO-10101), and data were collected on 2005 December 25. Details for these fields were presented in King et al. (2012). We independently re-reduced the two data sets from GO9444 and GO-10101 using the software described in Anderson et al. (2008). We then averaged the stellar magnitudes observed in the two data sets.

GO-9444 data were taken with an instrumental setup (GAIN=1), which does not allow us to recover the charge along the bleeding columns. Indeed, in that setup the digital saturation occurs before the physical one and the excess charge is irremediably lost. In contrast, the photometry of bright saturated stars was obtained from GO-10101 images, which were collected with an instrumental setup that allows the excess charge to be recovered (GAIN=2), as demonstrated by Gilliland (2004). For saturated stars, we used the positions and the fluxes provided by King et al. (2012) and derived by fitting the wings of the PSFs (see section 8.1 from Anderson et al. 2008 for details).

\footnotetext{
${ }^{1}$ Specifically, the short exposures were collected with a number of sampling equal to 15 (NSAMP=15), organized in linear sampling mode with steps of $10 \mathrm{~s}$ (SAMP-SEQ=SPARS10), while deep exposure with NSAMP $=14$ in mode SAMP-SEQ $=$ SPARS100. See WFC3 Instrument Handbook (for Cycle 25) http://www.stsci.edu/hst/wfc3/ documents/handbooks/currentIHB/wfc3_cover.html, section 7.7.3 MULTIACCUM timing sequences: full array apertures for details.

${ }^{2}$ http://www.stsci.edu/ $/$ jayander/WFC3/
} 
Table 1. Column (1) provides the progressive ID of the stars analysed in this paper. Columns (2) and (3) are the absolute equatorial position tightened to Gaia-DR1 system (Lindegren et al. 2016) in equinox J2000.0 at epoch 2015.5; columns (4) and (5) are the coordinate positions on a reference fits image also released with this paper. The full version of this table is available in the electronic version of the paper.

\begin{tabular}{lccccccccc}
\hline ID & RA & DEC & $X$ & $Y$ & $F 606 W$ & $F 814 W$ & $F 110 W$ & $F 160 W$ & $\begin{array}{c}\text { Member } \\
(10)\end{array}$ \\
$(1)$ & $(2)$ & $(3)$ & $(4)$ & $(5)$ & $(6)$ & $(7)$ & $\begin{array}{c}(9) \\
(10)\end{array}$ \\
\hline 1 & $13: 25: 29.191$ & $-47: 40: 57.59$ & 2100.66 & 1943.98 & 22.869 & 21.605 & 20.876 & 20.155 \\
2 & $13: 25: 28.842$ & $-47: 40: 56.46$ & 2151.75 & 1804.16 & 24.832 & 23.351 & 22.527 & 21.745 \\
3 & $13: 25: 29.137$ & $-47: 40: 56.19$ & 2157.97 & 1924.22 & 23.194 & 21.895 & 21.162 & 20.452 \\
4 & $13: 25: 28.924$ & $-47: 40: 55.82$ & 2176.15 & 1838.42 & 25.218 & 23.166 & 22.076 & 21.376 \\
5 & $13: 25: 28.920$ & $-47: 40: 51.92$ & 2332.99 & 1843.02 & 19.966 & 19.230 & 1 & 1 \\
\end{tabular}

\subsection{Calibration, proper motions and differential reddening}

Photometry has been calibrated into the Vega magnitude system by following the recipe of Bedin et al. (2005). For WFC/ACS photometry, we have used the zero-points from Bedin et al. (2005), while in the case of NIR photometry, we have adopted the zero-points provided by STScI web page for WFC3/NIR. ${ }^{3}$

Since the multiple MSs are the main target of this paper, we have analysed only relatively isolated stars, which are well fitted by the PSF model and have small magnitude and position rms. These stars have been selected by using the criteria described in detail by Milone et al. (2009) and Bedin et al. (2009). Briefly, for each star we have calculated a number of parameters that can be used as diagnostics of the photometric and astrometric qualities. These diagnostics include the photometric and astrometric rms, the fraction of light in the aperture due to neighbours (o), the quality of the PSF fit (q) and the excess of flux outside the PSF core (RADX). We plotted each parameter against the stellar magnitude and verified that most of the stars define a clear relation as a function of the magnitude. Outliers are stars with poor astrometric and photometric quality and are not considered in this paper. Photometry has been corrected for differential reddening by using the procedure described by Milone et al. (2012a). To estimate the amount of differential reddening associated with each star, we first derived the fiducial line of MS-I stars in the $m_{F 160 W}$ versus $m_{F 606 W}-m_{F 160 W}$ plane, which is the most sensitive CMD to differential reddening. Then, we calculated the residuals between the colour of each star and the colour of the fiducial along the reddening line. We assume as best estimate of the differential reddening associated with each star, the median of the residual values of the 75 neighbours MS-I stars. We refer to section 3.1 from Milone et al. (2012a) for further details.

We have used the relative proper motions of the stars in the analysed field of view to separate field stars from cluster members. Proper motions have been derived as in previous papers from our group (e.g. Anderson \& King 2003; Bedin et al. 2003) by comparing the distortion-corrected coordinates of stars derived from the GO-14118 and GO-9444 data sets, which provide the longest time baseline. We make public the astrometric and photometric catalogue of all sources studied in this paper. The first five lines of the catalogue are listed in Table 1 . We also release an astrometrized stacked image in the band $F 814 W$. Columns (6)-(9) are the calibrated magnitudes into the Vega-mag system $m_{F 606 W}, m_{F 814 W}, m_{F 110 W}, m_{F 160 W}$ (where the magnitude is not available for a given filter, the value is flagged to 99.9999); finally in column (10), we give a flag for proper-motion-based membership: 1 for members, and 0 for field objects.

\footnotetext{
${ }^{3}$ http://www.stsci.edu/hst/wfc3/phot_zp_lbn
}

\section{THE COLOUR-MAGNITUDE DIAGRAM}

The left-hand panel of Fig. 1 shows the $m_{F 814 W}$ versus $m_{F 606 W}-$ $m_{F 814 W}$ CMD for all the stars that pass the criteria of selection discussed in Section 2.3 and for which both proper motions and NIR photometry are available. The inset shows the vector-point diagram of the stellar displacements, DX and DY, in units of NIR/WFC3 pixel, while the $F 814 W$ magnitude is plotted as a function of the total displacement $\mathrm{DR}=\sqrt{\mathrm{DX}^{2}+\mathrm{DY}^{2}}$ in the middle panel of Fig. 1. The red line separates candidate cluster members (black points) from field stars (orange crosses). To derive this boundary, we have divided the magnitude range with $15.75<m_{F 814 W}<26.75$ into intervals of $0.25 \mathrm{mag}$ and for each of these we have derived the mean magnitude, and the median and the 68th percentile of the DR displacements of the cluster members (hereafter $\sigma_{\mathrm{DR}}$ ). The red line was derived by linearly interpolating the points with abscissa equal to four times $\sigma_{\mathrm{DR}}$ and ordinate corresponding to the mean magnitudes. The sample of cluster members used to derive the red line has been determined iteratively. At the first iteration, we used all the stars with $\mathrm{DR}<0.4$ pixel, while at the subsequent iterations we considered as candidate cluster members all the stars on the left-hand side of the red line. The procedure has been repeated three times until convergence.

A visual inspection of the CMD of cluster members plotted in the left-hand panel of Fig. 1 reveals that the blue and the red MSs of $\omega$ Centauri, discovered by Bedin et al. (2004), are clearly visible from the brightest magnitude before saturation down to an interval of about three magnitudes in the $F 814 W$ band. At fainter luminosity, $m_{F 814 W} \gtrsim 21.4$, the two distinct blue and the red MSs are not distinguishable and most of the cluster stars form a single and broadened MS. The only exception is provided by a third poorly populated red MS, previously identified by King et al. (2012), which includes only a few per cent of the total number of MS stars.

The NIR, $m_{F 160 W}$ versus $m_{F 110 W}-m_{F 160 W}$, CMD is plotted in the right-hand panel of Fig. 1. In this case, the blue and the red MSs are visible in the upper part of the CMD and merge together at the level of the MS knee around $m_{F 160 W} \sim 19.5$. A double MS is clearly visible at fainter magnitudes, where the more populated MS has bluer $m_{F 110 W}-m_{F 160 W}$ colours in contrast with what we observe above the MS knee, where the blue MS is less populated. Noticeably, below $m_{F 160 W} \sim 19.5$, most of the field stars follow the more populated MS of $\omega$ Centauri, suggesting peculiar properties for the stars in the less-populated component.

\section{THE COMPLEX MS OF $\omega$ CENTAURI}

In this section, we identify the multiple stellar populations along the MS. We first analyse the region of the CMD below the MS knee in Section 4.1, while in Section 4.2 we identify and investigate the 


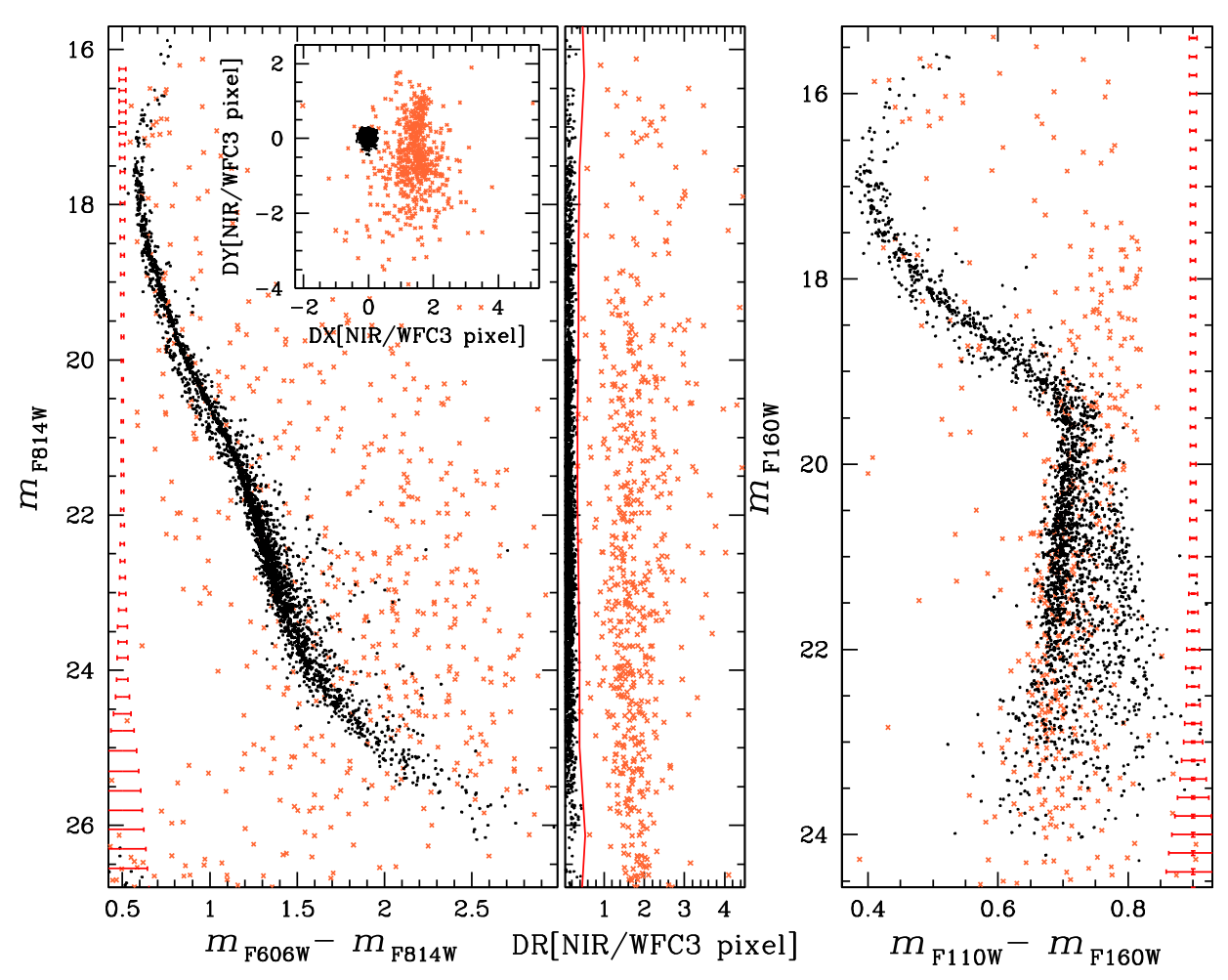

Figure 1. $m_{F 814 W}$ versus $m_{F 606 W}-m_{F 814 W}$ (left-hand panel) and $m_{F 160 W}$ versus $m_{F 110 W}-m_{F 160 W}$ (right-hand panel) CMD of stars in the NIR/WFC3 field for which proper-motion measurements are available. The inset in the left-hand panel shows the vector-point diagram of the relative stellar displacements. Middle panel shows $m_{F 814 W}$ as a function of the total displacement DR. The red line separates the probable cluster members from field stars, which are represented with black dots and orange crosses, respectively, in all the panels of the figure.

two main stellar populations of $\omega$ Centauri along the entire CMD, from the SGB towards the hydrogen-burning limit.

\subsection{Multiple populations at the bottom of the MS}

In order to identify multiple populations of M-dwarf stars, we start by extending the procedure introduced by Milone et al. (2015) in their study of the GC NGC 7089 (M2) to MS stars with $20.1<m_{F 160 W}<22.6$.

The procedure by Milone and collaborators is summarized in the following and illustrated in Fig. 2. Panel (a1) shows the $m_{F 160 W}$ versus $C_{F 606 W, F 814 W, F 160 W}=\left(m_{F 606 W}-m_{F 814 W}\right)-\left(m_{F 814 W}-m_{F 160 W}\right)$ pseudo-CMD of stars in the analysed interval of magnitude. The red and the blue lines are the blue and the red envelopes of the MS and have been derived as follows. We have first divided the $F 160 \mathrm{~W}$ magnitude interval in a series of magnitude bins of width $v=0.25 \mathrm{mag}$ by following the naive estimator method (Silverman 1986). The bins are defined over a grid of $N$ points separated by steps of fixed magnitude $(s=v / 5)$. For each bin, $i$, we have calculated the 5 th and the 95th percentile of the $m_{F 606 W}-m_{F 160 W}$ colour distribution $\left(x 1_{i}\right.$ and $x 2_{i}$ ) and associated these points with mean $m_{F 160 W}$ magnitude of the stars in the bin $\left(y_{i}\right)$. The points $\left(x 1_{i}, y_{i}\right)$ and $\left(x 2_{i}, y_{i}\right)$ were then smoothed to obtain the blue and the red lines shown in panel (a1) of Fig. 2, respectively. We have used the boxcar averaging smoothing, where each point has been replaced by the average of the three adjacent points.

We verticalized the CMD of the stars shown in panel (a1) by using the relation:

$\Delta_{1}=\left[\left(X-X_{\text {redfiducial }}\right) /\left(X_{\text {bluefiducial }}-X_{\text {redfiducial }}\right)\right]-1$, where $X=C_{F 606 W, F 814 W, F 160 W}$. We show $m_{F 160 W}$ versus $\Delta_{1}$ in panel (a2) of Fig. 2.

We derived the quantity $\Delta_{2}$ similarly, but by using the $m_{F 160 W}$ versus $m_{F 110 W}-m_{F 160 W}$ diagram shown in panel (b1) of Fig. 2. The magnitude $m_{F 160 W}$ is plotted against $\Delta_{2}$ in panel (b1). In panel (c1) of Fig. 2, we show the $\Delta_{2}$ versus $\Delta_{1}$ diagram for the analysed MS stars of $\omega$ Centauri, while panel (c2) shows the corresponding Hess diagram. This diagram is similar to the one defined by Milone et al. $(2015,2017)$ and will be indicated as 'chromosome map' by following the nickname by Renzini et al. (2015). All the panels of Fig. 2 show the same stars.

The red points plotted in the bottom-left corner of panel (c1) represent the distribution of stars that we would expect from observational errors only and are randomly extracted from a Gaussian distribution where the dispersion corresponds to the uncertainties. The fact that the observed $\Delta_{1}$ and $\Delta_{2}$ distribution of stars is significantly wider than the corresponding error distribution proves that the chromosome map of $\omega$ Centauri is not consistent with a simple population. A visual inspection of the figures in panels (c1) and (c2) suggests that four main bumps are present along this sequence that we indicate as A, B, C and D and colour orange, yellow, cyan and blue, respectively, in the $\Delta_{2}$ versus $\Delta_{1}$ diagram shown in panel (c3).

This diagram reveals two additional poorly populated sequences of stars that span the interval of $-0.2 \lesssim \Delta_{2} \lesssim 1.0$ and we name them $S_{1}$ and $S_{2}$. They are represented with aqua and magenta starred symbols, respectively, and, as we will see in Section 4.2, are well separated in the $m_{F 814 W}$ versus $m_{F 606 W}-m_{F 160 W}$ CMD.

To demonstrate that the selected A-D stellar groups are consistent with four stellar populations, we compare in Fig. 3 the observed 


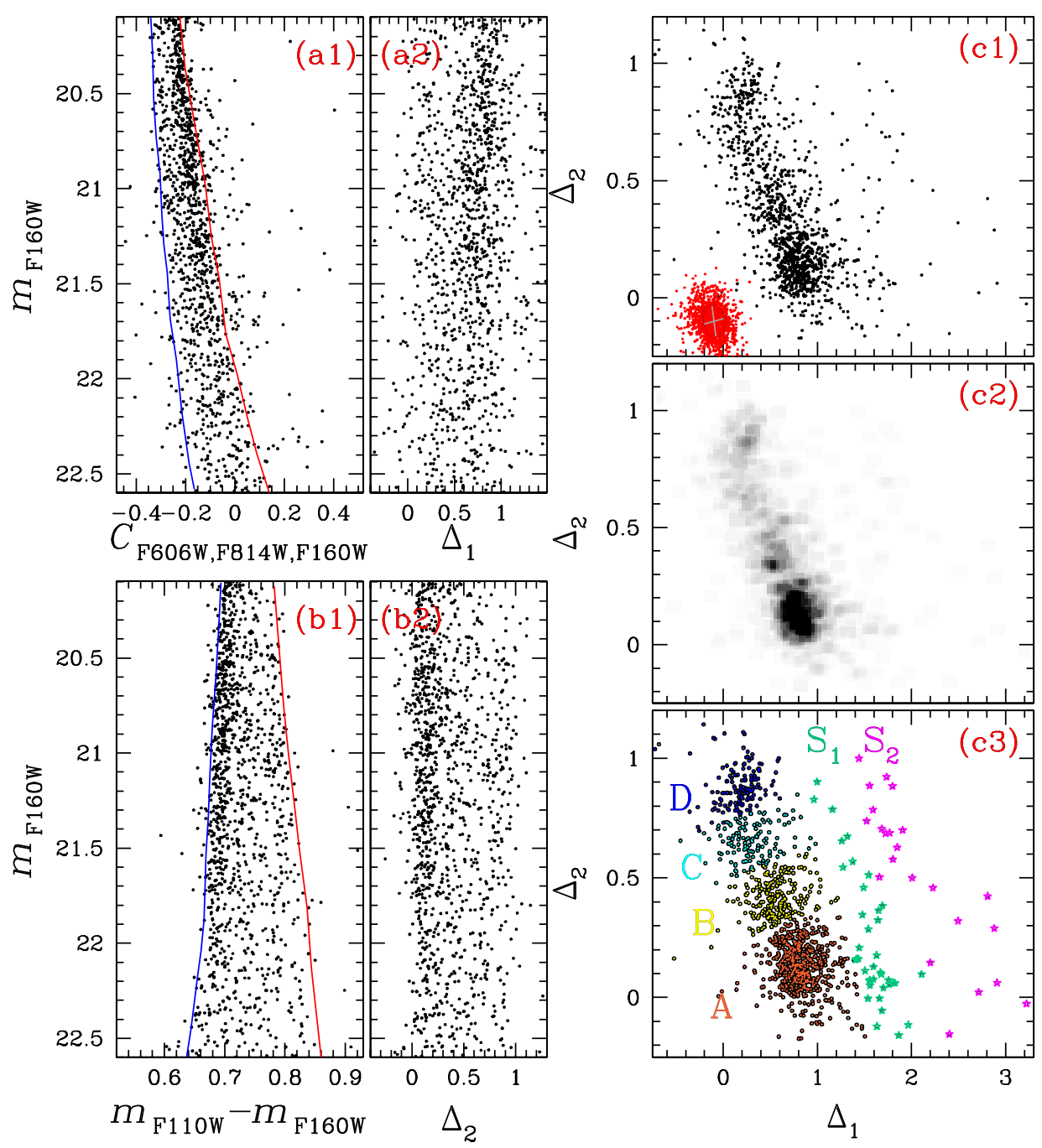

Figure 2. Panels (a1) and (b1) show the $m_{F 160 W}$ versus $C_{F 606 W, F 814 W, F 160 W}$, and $m_{F 160 W}$ versus $m_{F 110 W}-m_{F 160 W}$ diagram, respectively, for MS stars fainter than the MS knee. The red and the blue lines superimposed on each diagram represent the fiducial lines used to derive the corresponding verticalized $m_{F 160 W}$ versus $\Delta_{1}$ and $m_{F 160 W}$ versus $\Delta_{2}$ diagrams plotted in panels (a2) and (b2). The $\Delta_{2}$ versus $\Delta_{1}$ pseudo-two-colour diagram or 'chromosome map' is plotted in panels (c1) and (c3), while panel (c2) shows the corresponding Hess diagram. The red points indicate the stellar distribution expected from observational errors only, where the red ellipses include the 68.27 per cent of points. In the chromosome map in panel (c3), we have represented stars of populations A, B, $\mathrm{C}, \mathrm{D}, \mathrm{S}_{1}$ and $\mathrm{S}_{2}$ with orange, yellow, cyan, blue, aqua and magenta symbols, respectively (see text for details).

chromosome map of $\omega$ Centauri stars (panel a1) and the chromosome map of four simple stellar populations. We named these populations $\mathrm{A}_{\mathrm{SIM}}, \mathrm{B}_{\mathrm{SIM}}, \mathrm{C}_{\mathrm{SIM}}$ and $\mathrm{D}_{\mathrm{SIM}}$, and coloured orange, yellow, cyan and blue, respectively, in panel (a2) of Fig. 3. We assumed that all the simulated stars in the population $\mathrm{A}_{\text {SIM }}$ are clustered around the average $\Delta_{1}$ and $\Delta_{2}$ values of the observed population A stars and adopted similar criteria for the other three groups of simulated stars. The $\Delta_{1}$ and $\Delta_{2}$ scatter around the average value that is visible in panel (a2) of Fig. 3 is due to observational errors. Simulations shown in panel (a2) are obtained by randomly extracting points from a two-dimensional Gaussian distribution, where the dispersion corresponds to the observed uncertainties.

To compare the observed and the simulated stars, we first arbitrarily selected the grey dot and drew the straight line shown in panels (a1) and (a2) of Fig. 3. Then we translated and rotated all the stars of panels (a1) and (a2) in such a way that the origin of the new reference frame corresponds to the grey dot and the grey line translates into a vertical line with ordinate equal to zero. The derived $\Delta_{2}^{\prime}$ versus $\Delta_{1}^{\prime}$ diagram is plotted in panels (b1) and (b2) for observed and simulated stars, respectively.

Panel (c1) of Fig. 3 shows the $\Delta_{2}^{\prime}$ histogram diagram for observed populations A-D stars while the corresponding kernel-density distribution, derived by using a Gaussian kernel with dispersion of $0.02 \mathrm{mag}$, is represented with the red dash-dotted line. The $\Delta_{2}^{\prime}$ histogram distribution for simulated stars is plotted in panel (c2). Both the histogram distributions and the kernel-density distribution for simulated (blue line) and observed stars (red dash-dotted line) are quite similar. This fact demonstrates that the four groups, A-D, of stars are actually consistent with four stellar populations.

Similarly, we note that the $\Delta_{1}$ and $\Delta_{2}$ distribution of $S_{1}$ and $S_{2}$ stars is significantly wider than what we expect from observational errors only, thus indicating that each sequence hosts more than one stellar population.

A visual inspection of the chromosome maps shown in panels (a1) and (a2) reveals that the populations A-D are more clearly separated in the simulated diagram than in the observed ones. This fact 

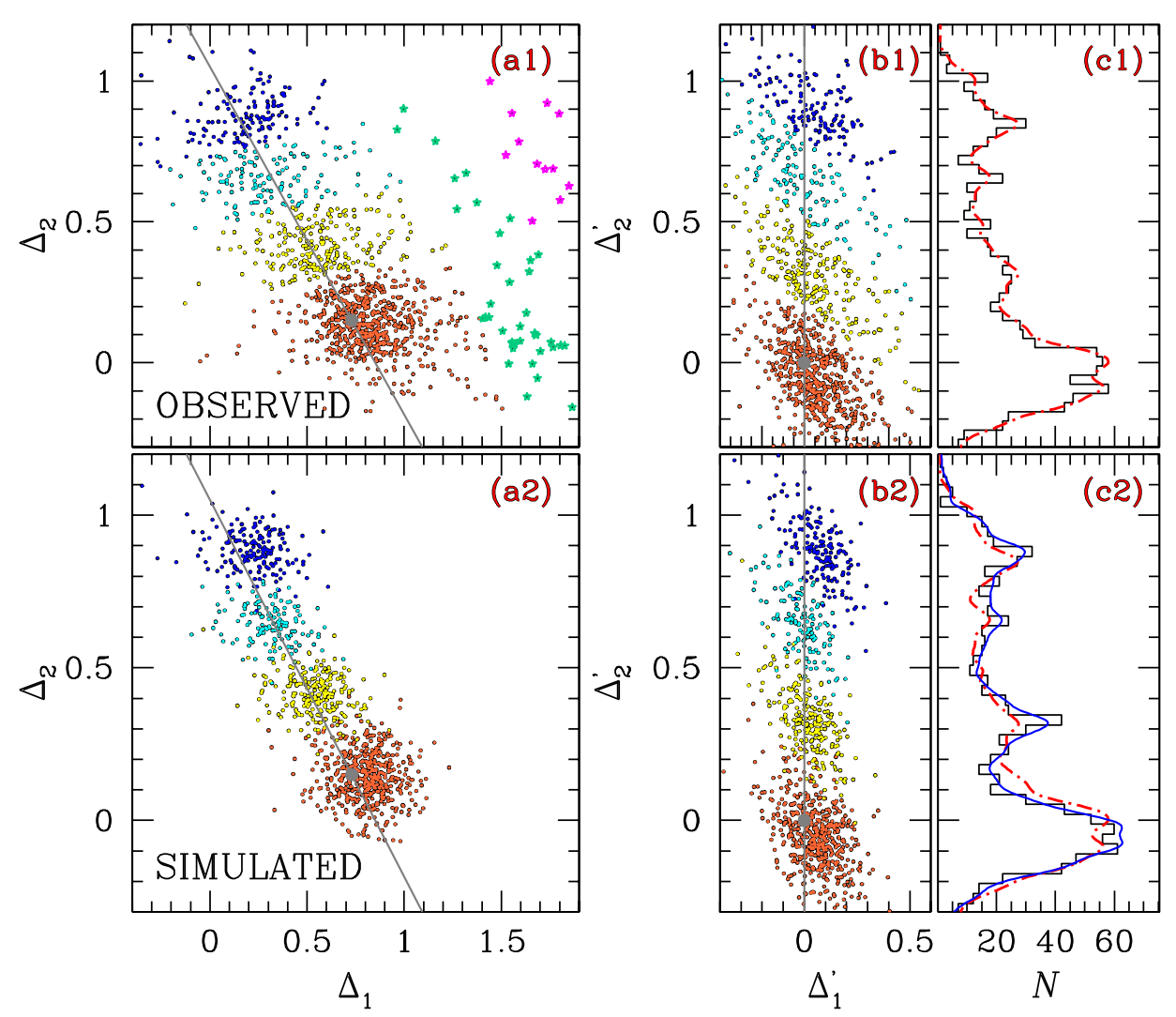

Figure 3. Reproduction of the chromosome map for the observed $\omega$ Centauri stars plotted in panel (c3) of Fig. 2 (panel a1) and simulated chromosome map of four stellar populations (panel a2). Panels (b1) and (b2) show the corresponding verticalized $\Delta_{2}^{\prime}$ versus $\Delta_{1}^{\prime}$ diagrams, while the histogram distribution of the $\Delta_{2}^{\prime}$ quantities for observed populations A-D and simulated stars are plotted in panels (c1) and (c2), respectively. The $\Delta_{2}^{\prime}$ kernel-density distribution of the observed stars is represented with red dash-dotted line in both panels (c1) and (c2), while the blue line shown in panel (c2) marks the corresponding distribution for simulated stars (see text for details).

is quite expected. Indeed, it is well known from literature studies on the chromosome map of $\omega$ Centauri RGB stars that this cluster exhibits at least 15 stellar populations (Milone et al. 2017, see also Bellini et al. 2010, Marino et al. 2011 and Villanova et al. 2014). This suggests that each group of populations A-D may host subpopulations of stars with different chemical composition that contribute to the $\Delta_{2}$ versus $\Delta_{1}$ broadening of each population.

\subsection{The two main stellar populations of $\omega$ Centauri}

To further investigate the multiple stellar populations along the MS, we show in Fig. 4 two CMDs and a pseudo-CMD 4 of $\omega$ Centauri stars obtained from different combinations of colours and magnitudes. In each diagram, the two most evident MSs are well separated only at specific and well-defined magnitude intervals. Specifically, in the $m_{F 160 W}$ versus $m_{F 606 W}-m_{F 160 W}$ and the $m_{F 160 W}$ versus $m_{F 110 W}-m_{F 160 W} \mathrm{CMDs}$, the two main populations are clearly visible in the upper and the lower MS, respectively. In the $m_{F 160 W}$ versus $\left(m_{F 606 W}-m_{F 814 W}\right)-\left(m_{F 110 W}-m_{F 160 W}\right)$ pseudo-CMD, the split MS is visible at intermediate luminosity only.

In order to identify the two main populations of $\omega$ Centauri along the entire MS, we have combined information from the

\footnotetext{
${ }^{4}$ We indicate as pseudo-CMD a diagram, like $\left(m_{F 606 W}-m_{F 814 W}\right)-$ $\left(m_{F 110 W}-m_{F 160 W}\right)$, where the ordinate corresponds to the stellar magnitude and the abscissa to a colour difference.
}

three diagrams of Fig. 4. We have first selected by eye from the left-hand panel CMD a sample of MS-I and MS-II stars in the magnitude range $17.30 \leq m_{F 160 W}<19.35$, where the two populations are clearly separated. Similarly, the middle panel pseudoCMD has been used to select MS-I and MS-II stars with 19.35 $\leq m_{F 160 W}<20.10$.

The separation between the two main MSs is less evident in the NIR CMD plotted in the right-hand panel of Fig. 4. Nevertheless, the majority of the stars populate the bluest MS component which is clearly connected with MS-I stars identified in the upper MS. Conversely, the remaining MS stars are distributed towards the red.

We note that in the magnitude range $20.10 \leq m_{F 160 W}<22.60$, the most populated MS hosts most of the population A and B stars identified in Fig. 2, while population-C and -D stars mostly correspond to the other MS component. In this magnitude interval, we will thus associate the sample of population-A and -B stars to the MS-I, and population-C and -D stars to the MS-II. At fainter magnitudes, we consider as MS-I and MS-II stars all the stars with $m_{F 110 W}-m_{F 160 W}$ greater than or smaller than 0.38 mag, respectively. The selected MS-I and MS-II stars are coloured blue and red, respectively, in the insets of Fig. 4.

The $m_{F 160 W}$ versus $m_{F 606 W}-m_{F 160 W}$ CMD shown in the bottomleft panel of Fig. 4 reveals that the MS-I and the MS-II clearly correspond to the red and the blue MSs discovered by Anderson (1997) and Bedin et al. (2004). A third, poorly populated MS that exhibits redder $m_{F 606 W}-m_{F 160 W}$ and $m_{F 814 W}-m_{F 160 W}$ colours than the majority of MS stars with the same $F 160 \mathrm{~W}$ magnitude, is 


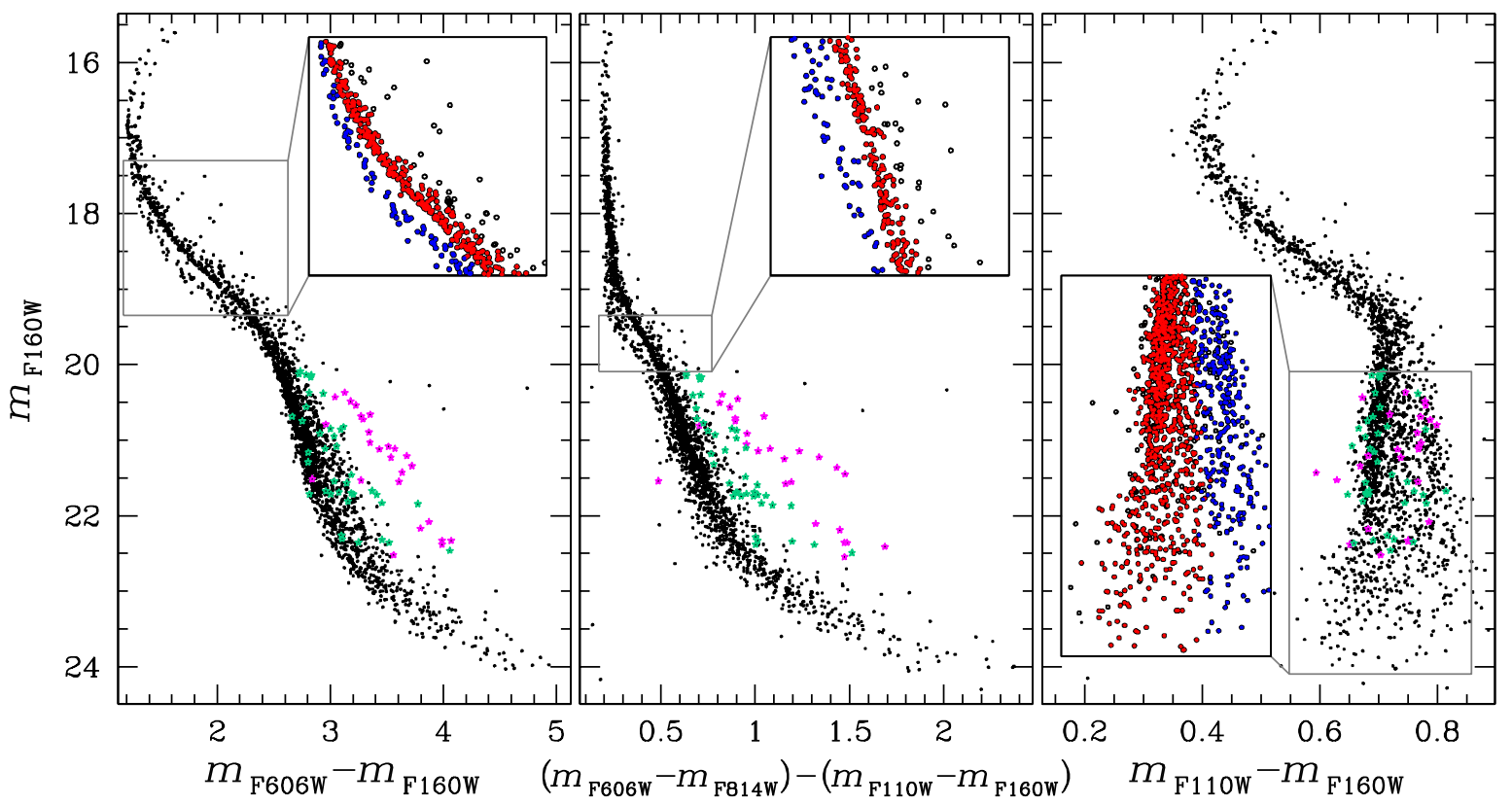

Figure 4. $m_{F 160 W}$ versus $m_{F 606 W}-m_{F 160 W} \mathrm{CMD}$ (left), $m_{F 160 W}$ versus $\left(m_{F 606 W}-m_{F 814 W}\right)-\left(m_{F 110 W}-m_{F 160 W}\right)($ middle $)$ and $m_{F 160 W}$ versus $m_{F 110 W}-m_{F 160 W}$ CMD (right) of $\omega$ Centauri stars. The insets are zoom of the corresponding diagrams where the two MSs are clearly visible. Red and blue colours indicate MS-I and MS-II stars, respectively. $\mathrm{S}_{1}$ and $\mathrm{S}_{2}$ stars identified in Fig. 2 are coloured aqua and magenta, respectively.

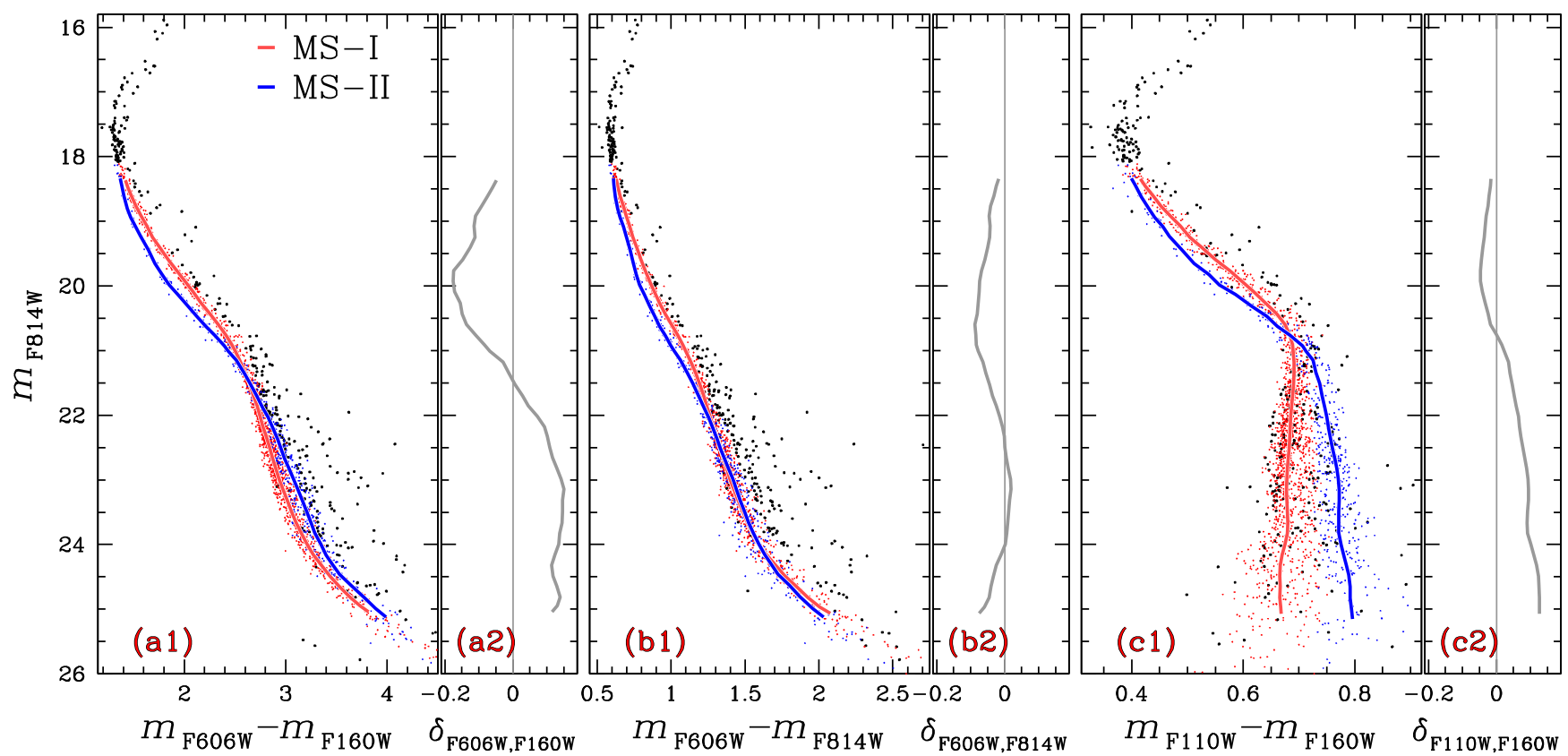

Figure 5. Collection of optical and NIR CMD of $\omega$ Centauri of Fig. 1. The sample of MS-I and MS-II stars identified in Fig. 4 are coloured red and blue, respectively, in panels (a1), (b1) and (c1), while the continuous lines are the corresponding fiducial lines. In panels (a2), (b2) and (c2), we have represented with thick grey lines the $F 814 \mathrm{~W}$ magnitude as a function of the colour difference between the blue and red fiducial lines (see text for details).

visible below $m_{F 160 W} \sim 20.5$ and corresponds to the sequence $S_{2}$ identified in Fig. 2. $S_{1}$ stars have intermediate $m_{F 606 W}-m_{F 160 W}$ and $m_{F 814 W}-m_{F 160 W}$ colours. Noticeably both $\mathrm{S}_{1}$ and $\mathrm{S}_{2}$ are spread over a relatively large interval of $m_{F 110 W}-m_{F 160 W}$.

Finally we remark that, as previously shown by King et al. (2012), the SGB is clearly bimodal in each CMD of Fig. 4, with the bright SGB hosting the majority of SGB stars. Below we will show that the bright and faint SGBs are the progeny of MS-I and MS-II stars, respectively.
In Fig. 5, we have used red and blue colours to represent the MS-I and MS-II stars, respectively, identified in Fig. 4 in three CMDs made by plotting the $F 814 W$ magnitudes as a function of three different colours. Specifically, we use in panel (a1) the widest colour baseline, $m_{F 606 W}-m_{F 160 W}$, while in panels (b1) and (c1) we show the optical, $m_{F 606 W}-m_{F 814 W}$, and NIR, $m_{\mathrm{F} 110 \mathrm{~W}}-m_{\mathrm{F} 160 \mathrm{~W}}$, colours, respectively.

We immediately note that the two main populations can be followed continuously from the turn-off towards the hydrogen-burning 


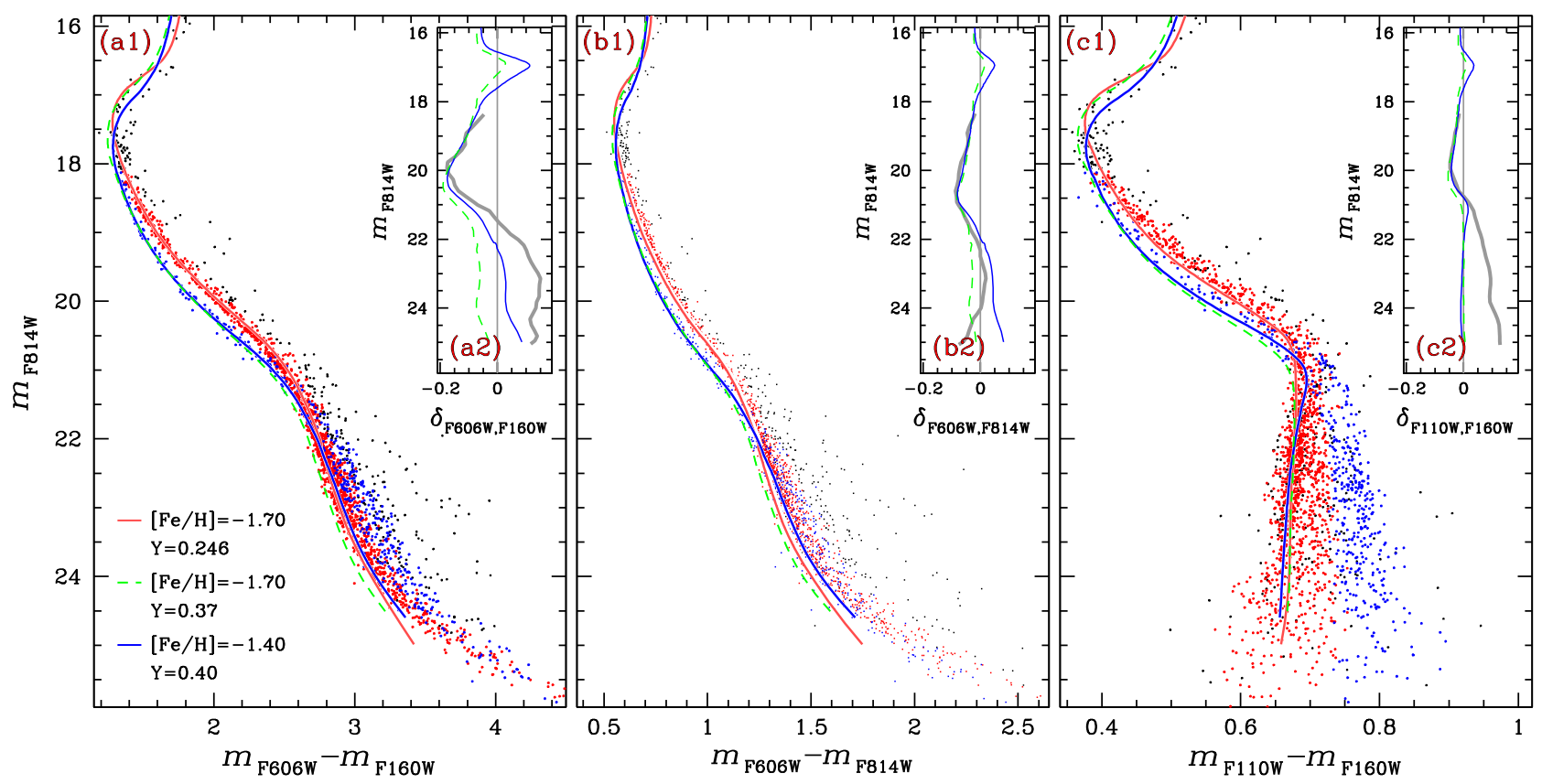

Figure 6. Comparison between the observed CMDs of Fig. 5 and isochrones from Dotter et al. (2008). MS-I and MS-II stars are coloured red and blue, respectively. The red isochrones correspond to the stellar population with primordial helium abundance and $[\mathrm{Fe} / \mathrm{H}]=-1.70$. The blue-continuous and greendashed isochrones correspond to the helium-enhanced stellar populations with $[\mathrm{Fe} / \mathrm{H}]=-1.40$ and -1.70 , respectively (see text for details). In the insets (a2), (b2) and (c2), we have compared the colour difference between each isochrone and the metal-poor isochrone and the corresponding colour difference between the fiducial of the MS-II and that of the MS-I.

limit, thus demonstrating that the groups of MS-I and MS-II stars identified in the distinct magnitude intervals of Fig. 4 actually trace the same stellar population along the entire CMD. The red and the blue lines overimposed on each diagram are the fiducial lines of the two main populations and have been derived by using a procedure based on the naive estimator method (Silverman 1986) described in Section 4.1.

We also calculated the $m_{F 606 W}-m_{F 160 W}$ colour difference, $\delta_{F 606 W, F 160 W}$, between the blue and the red fiducial lines and we show $m_{F 814 W}$ against $\delta_{F 606 W, F 160 W}$ in panel (a2). We obtained $\delta_{F 606 W, F 814 W}$ and $\delta_{F 110 W, F 160 W}$ similarly and plot $m_{F 814 W}$ as a function of these quantities in panels (b2) and (c2).

In the upper part of the CMD, MS-I is redder than MS-II in all the analysed CMDs, and the colour separation increases when moving from the Main Sequence Turn Off towards fainter magnitudes and has a maximum at $m_{F 814 W} \sim 20.0$. Below that, the MS-I and the MS-II get closer and merge together at the luminosity of the MS knee, around $m_{F 814 W} \sim 21.0$.

The MS-II is redder than the MS-I at fainter magnitudes in both the $m_{F 606 W}-m_{F 160 W}$ and $m_{F 110 W}-m_{F 160 W}$ colour. Above the MS knee, the separation between the two MSs is maximum in the $m_{F 606 W}-m_{F 160 W}$ colour, where it approaches $\sim 0.17$ mag, and decreases for shorter colour baselines. At the bottom of the MS, the dependence of the MS separation from the colour baseline is less evident and, in each of these two CMDs, it is close to $0.12 \mathrm{mag}$ at $m_{F 814 W} \sim 24.0$. In the optical CMD, the two MSs are nearly coincident in the magnitude interval $22.5 \lesssim m_{F 814 W} \lesssim 24.0$ while MS-I is redder than MS-II at fainter luminosity.

\section{COMPARISON WITH THEORY}

To get information on the main populations of $\omega$ Centauri, we compare in Fig. 6 the observed CMDs with $\alpha$-enhanced $([\alpha / \mathrm{Fe}]=0.3)$ isochrones from Dotter et al. (2008). The red isochrones correspond to a stellar population with $[\mathrm{Fe} / \mathrm{H}]=-1.70$ and primordial helium abundance $Y=0.246$, while the blue isochrone has $[\mathrm{Fe} / \mathrm{H}]=-1.40$ and $Y=0.40$. The adopted metallicities have been selected in order to match the observed CMD. We also show with green dashed lines the isochrones with $Y=0.37$ and $[\mathrm{Fe} / \mathrm{H}]=-1.70$. We have assumed a distance modulus $(m-M)_{0}=13.69$ and a reddening $E(B-V)=0.13$, which are similar to the values listed by the Harris (1996, updated as in 2010) catalogue, and an age of 13.5 Gyr. The parameters corresponding to the best-fitting isochrones have been determined as in Dotter et al. (2010). Briefly, we started by setting the distance modulus, and reddening to the values provided by the 2010 version of the Harris (1996) catalogue. We assumed for the MS-I a metallicity $[\mathrm{Fe} / \mathrm{H}]=-1.75$ corresponding to the peak of the metallicity distribution derived from high-resolution spectroscopy by Marino et al. (2011). Moreover, we assumed that the MS-II is enhanced in $[\mathrm{Fe} / \mathrm{H}]$ by 0.3 dex with respect to the MS-I $\omega$ Centauri as inferred by Piotto et al. (2005). We have then adjusted the values of metallicity, reddening and distance to optimize the fit with stars brighter than the MS knee. The age has been then estimated by selecting the isochrone providing the best match with the position and shape of the upper SGB.

To better compare the relative colours of MS-I and MS-II stars with those of the isochrones, we have calculated the colour difference between each isochrone and the isochrone with $[\mathrm{Fe} / \mathrm{H}]=$ -1.70 and primordial helium abundance. In panels (a2), (b2) and (c2) of Fig. 6, we plot $m_{F 814 W}$ against the colour difference, $\delta$, derived from the isochrones (blue continuous and green dashed lines) and the corresponding colour difference between MS-II and MS-I shown in Fig. 5.

We emphasize here that, according to studies based on highresolution spectroscopy, stars in $\omega$ Centauri exhibit a complex metallicity distribution (e.g. Norris \& Da Costa 1995; Johnson \& 
Pilachowski 2010; Marino et al. 2011). Therefore, our choice of using only few isochrones is meant to reproduce the main features of the cluster CMD and the average properties of the two main populations of $\omega$ Centauri, and we refer to the paper by Tailo et al. (2016) for a more complete comparison between the upper part of the observed CMD of $\omega$ Centauri and theoretical isochrones. Nevertheless, we note that the value of $[\mathrm{Fe} / \mathrm{H}]$ that we have adopted for the MS-I roughly matches the main peak of the metallicity distribution derived by Marino et al. (2011) and the metallicity difference between the two isochrones is close to the average $[\mathrm{Fe} / \mathrm{H}]$ difference of red-MS and blue-MS stars derived by Piotto et al. (2005).

Fig. 6 reveals that the three isochrones reproduce well the CMD region above the MS knee. Specifically, the metal-poor and heliumpoor isochrones match the MS-I, and the helium-rich isochrones provide a good fit to the MS-II. Noticeably, the helium content needed for the metal-poor isochrone to match the MS-II is $Y=$ 0.37 , which is significantly lower than the helium abundance of the metal-rich isochrone $(Y=0.40)$. The helium-rich population of $\omega$ Centauri has been interpreted as a second stellar generation(s) formed by the ejecta of evolved intermediate-mass first-population stars (see e.g. D'Antona et al. 2011, 2016). In particular, the presence of metal-rich stars with helium abundance of $Y=0.40$ is consistent with the scenario suggested by Karakas, Marino \& Nataf (2014) where stars with extreme helium content formed from the ejecta of a previous generation of helium-rich stars.

The three isochrones qualitatively reproduce the shape of the two main SGBs, with the faint and the bright SGB corresponding to the helium-rich and helium-poor populations, respectively. These results are quite expected as the same conclusion has been obtained in several previous papers (e.g. Bedin et al. 2004; Norris 2004; Piotto et al. 2005; King et al. 2012; Tailo et al. 2016). In addition, we note that the MS-II is also consistent with a stellar population with the same metallicity as the MS-I but with different helium abundance.

Below the MS knee, the adopted isochrones overlap the MS-I and none of them reproduce the colours of MS-II stars. In particular, in the $m_{F 814 W}$ versus $m_{F 110 W}-m_{F 160 W} C M D$, the colour separation between the metal-rich isochrones and the metal-poor ones is smaller than $0.03 \mathrm{mag}$ for $m_{F 814 W}>21.0$. This fact indicates that, according to isochrones by Dotter et al. (2008), in the analysed interval of $\mathrm{He}$ and $\mathrm{Fe}$ abundances, the $m_{F 110 \mathrm{~W}}-m_{F 160 \mathrm{~W}}$ colour of faint M-dwarfs is marginally affected by variations in helium and iron content.

In this context, it is worth noting that variations in metallicity alone and variations in the $\mathrm{C}+\mathrm{N}+\mathrm{O}$ sum of $\omega$ Centauri stars alone are not viable hypotheses to explain the observed behaviour of MS-I and MS-II. The fact that the MS-II is more metal rich than the MS-I was clearly demonstrated by Piotto et al. (2005) on the basis of direct spectroscopic measurements of iron abundance in bright MS-I and MS-II stars. Since the MS-I is redder than the MS-II above the MS knee, Piotto and collaborators noticed that the only way to reproduce the colours and the metallicity of the two MSs of $\omega$ Centauri is to assume that the MS-II is also highly helium enhanced with respect to the MS-I. In this work, we do not take into account the possibility that the MS-I stars have higher metallicity than MS-II and refer to papers by Piotto et al. (2005), Bellini et al. (2010), Tailo et al. (2016) and references therein for photometric and spectroscopic metallicity determination of MS-I and MS-II stars. Similarly, we note that difference in the overall $\mathrm{C}+\mathrm{N}+\mathrm{O}$ alone is not able to reproduce the difference in colour between MS-I and MS-II stars that we measured in this paper. Indeed, a $\mathrm{C}+\mathrm{N}+\mathrm{O}$ variation produces a marginal colour difference for MS stars (e.g. Cassisi et al. 2008; Ventura et al. 2009; Sbordone et al. 2011).

\subsection{The effect of light elements on the colours of MS stars}

To further investigate the physical reasons responsible for the multiple sequences, we have extended the method introduced in our previous papers to $\omega$ Centauri and compared the colours of the observed sequences with those predicted by appropriate synthetic spectra (see Milone et al. 2012b, 2014 for details). Briefly, we first identified a series of reference points along the MS-I fiducial line with $F 814 \mathrm{~W}$ magnitude ranging from 17.5 to 24.0 in steps of $0.5 \mathrm{mag}$. For each point, we used the best-fitting isochrone of MS-I stars to derive the corresponding values of effective temperature $\left(T_{\text {eff }}\right)$ and gravity $(\log g)$. For each reference point, we simulated two spectra with distinct $\mathrm{C}, \mathrm{N}, \mathrm{O}$ abundances. For the first spectrum (reference spectrum), we assumed $[\mathrm{C} / \mathrm{Fe}]=0.4,[\mathrm{~N} / \mathrm{Fe}]=0.2$ and $[\mathrm{O} / \mathrm{Fe}]=$ 0.3 , while for the second one (comparison spectrum), we adopted $[\mathrm{C} / \mathrm{Fe}]=-0.5,[\mathrm{~N} / \mathrm{Fe}]=1.1$ and $[\mathrm{O} / \mathrm{Fe}]=-0.5$. The adopted $\mathrm{C}$, $\mathrm{N}, \mathrm{O}$ abundances match the abundances derived for these elements by Marino et al. (2011, 2012) and Johnson \& Pilachowski (2010).

These chemical abundances and values of $T_{\text {eff }}$ and $\log g$ have been adopted as input parameters for the ATLAS 12 and SYNTHE codes (Castelli 2005; Kurucz 2005; Sbordone, Bonifacio \& Castelli 2007) to generate a grid of synthetic spectra in the wavelength range between 4500 and $17500 \AA$. We have included the following molecules in the Kurucz compilation: $\mathrm{CO}, \mathrm{C}_{2}, \mathrm{CN}, \mathrm{OH}, \mathrm{MgH}, \mathrm{SiH}$, $\mathrm{H}_{2} \mathrm{O}, \mathrm{TiO}, \mathrm{VO}, \mathrm{ZrO}$ (Partridge \& Schwenke 1997; Schwenke 1998; B. Plez, private communication). The resulting spectra for two stars, below and above the knee, are illustrated in Fig. 7, and correspond to MS stars with $m_{F 814 W}=23.25$ and 20.75, respectively. Upper panels compare the reference and the comparison spectra, while lower panels show the flux ratio between the two spectra as a function of the wavelength. We note that low-temperature spectra with different chemical composition are very different along most of the analysed wavelength range, whereas the two hot spectra are very similar. In particular, we confirm previous findings that the strong flux difference of M-dwarfs in the spectral region with $\lambda \gtrsim 14000 \AA$ is mostly due to oxygen abundance in the atmospheres of MS-I and MS-II stars through the absorption of the $\mathrm{H}_{2} \mathrm{O}$ molecules (Milone et al. 2012b; 2014; Dotter et al. 2015).

The synthetic spectra have been integrated over the transmission curves of the ACS/WFC and WFC3/NIR filters used in this paper to derive synthetic magnitudes. For each reference point, we have then calculated the difference between the $F 606 W, F 814 W, F 110 W$ and $F 160 \mathrm{~W}$ magnitudes obtained from the comparison and the reference spectrum (dmag).

We confirm the previous finding that, above the knee, the effect of $\mathrm{C}, \mathrm{N}, \mathrm{O}$ variations is negligible when both the optical and NIR colours are used (e.g. Sbordone et al. 2011; Dotter et al. 2015; Milone 2015). In contrast, the effect of light elements strongly affects the flux of fainter stars than the knee stars in the optical and NIR bands used in this paper.

The blue and green isochrones plotted in Fig. 8 have been obtained by adding to the helium-rich isochrones the corresponding values of dmag. These isochrones provide a qualitatively better fit of the entire CMD, from the SGB to the bottom of the explored MS.

\subsection{The stellar subpopulations of $\omega$ Centauri}

The six subpopulations identified in Section 4.1 are visible over a small magnitude interval below the MS knee. To constrain their 

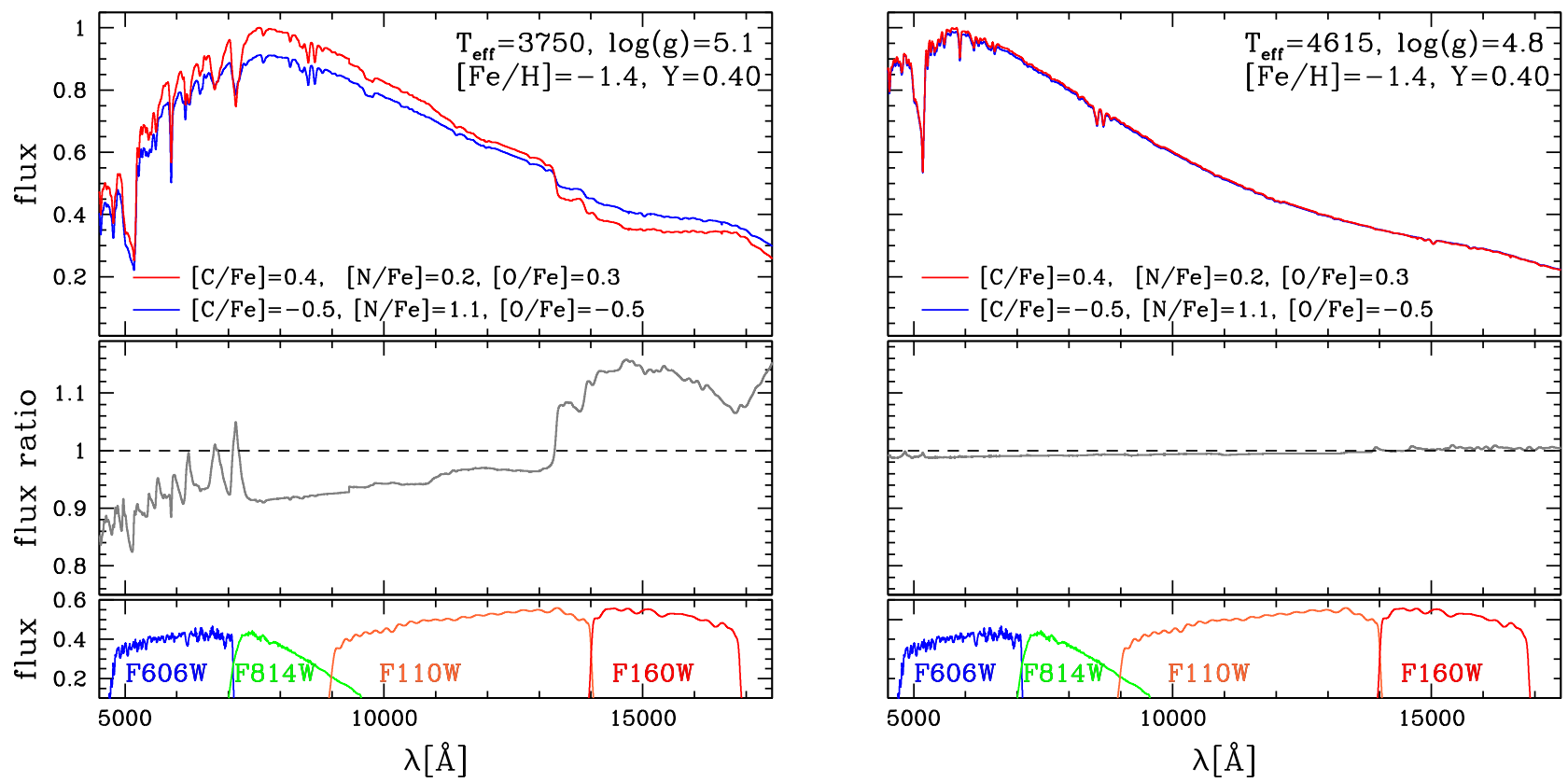

Figure 7. Synthetic spectra for an MS star with $[\mathrm{Fe} / \mathrm{H}]=-1.4$ and $Y=0.40$ located below (left-hand panels) and above (right-hand panels) the MS knee. Upper panels compare a blue and a red spectrum, named comparison and reference spectrum, respectively, with the same effective temperature and gravity but different chemical composition (see text for details). Middle panels show the ratio between the flux of the comparison and the reference spectrum as a function of the wavelength, while in lower panels we plot the transmission curves of the ACS/WFC and NIR/WFC3 spectra used in this paper.
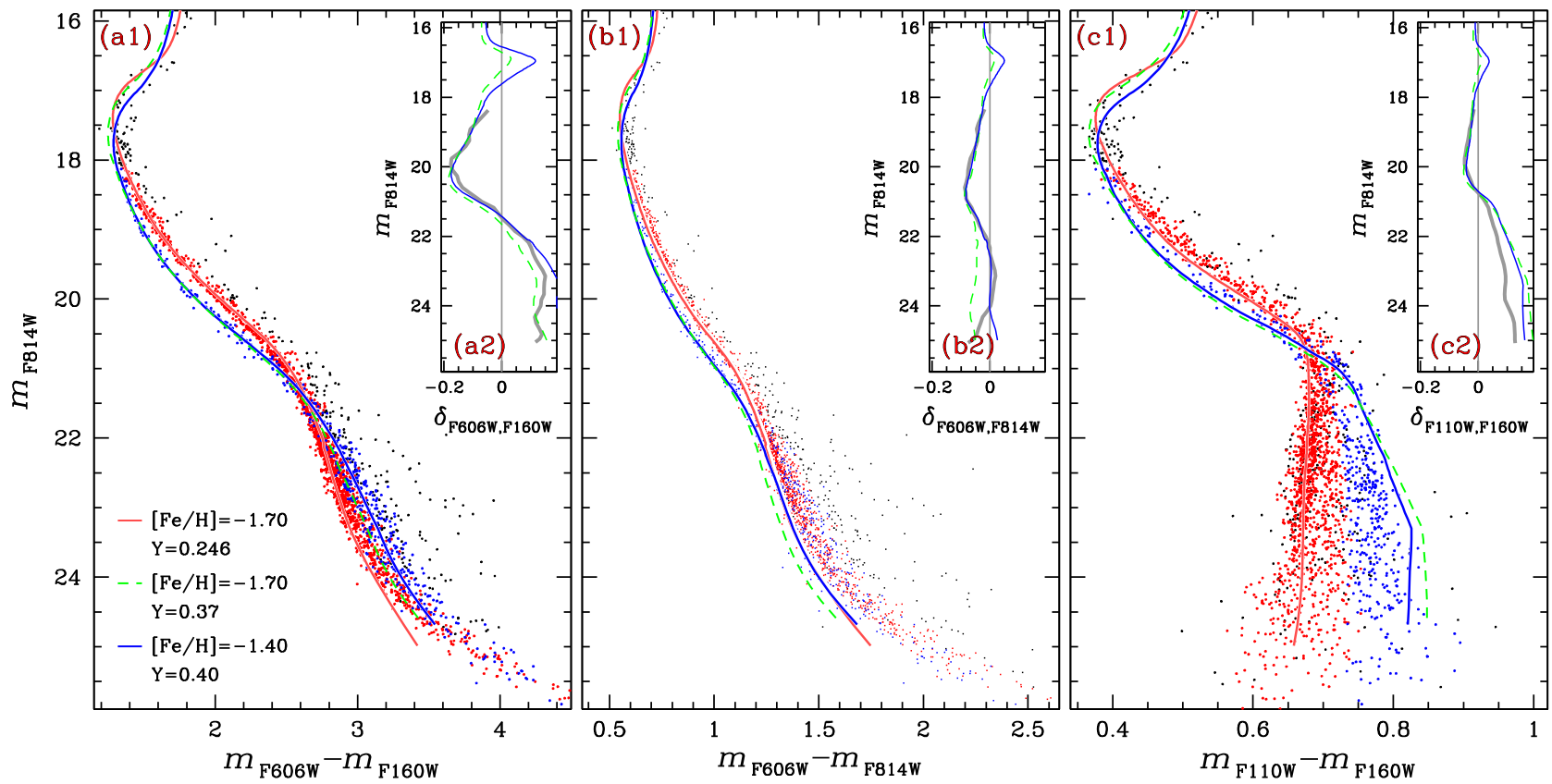

Figure 8. As in Fig. 6, but in this case the blue and green isochrones account for the variation of C, N, O typical of second-population stars in $\omega$ Centauri (see text for details).

main properties, we combine the observations of this paper with isochrones from Dotter et al. (2008) and literature results.

In the upper panels of Fig. 9, we reproduce the three CMDs of Fig. 5 and mark $S_{1}$ and $S_{2}$ stars with aqua and magenta starred symbols, respectively. We note that both subpopulations $S_{1}$ and $S_{2}$ have larger $m_{F 606 W}-m_{F 160 W}$ and $m_{F 606 W}-m_{F 814 W}$ colours than the majority of MS stars with the same $m_{F 814 W}$ luminosity. In contrast, $S_{1}$ and $S_{2}$ stars are mostly overimposed to the remaining MS stars in the $m_{F 814 W}$ versus $m_{F 110 W}-m_{F 160 W} \mathrm{CMD}$. On average, $\mathrm{S}_{2}$ stars are redder than $\mathrm{S}_{1}$ stars with similar $F 814 W$ magnitude level in all the CMDs of Fig. 9.

High-resolution spectroscopy reveals that $\omega$ Centauri hosts stellar populations which are highly iron enhanced with respect to the majority of $\omega$ Centauri stars $([\mathrm{Fe} / \mathrm{H}] \gtrsim-1.4$, e.g. Norris \& Da Costa 1995; Johnson et al. 2009). The most metal-rich stars have $[\mathrm{Fe} / \mathrm{H}]=\sim-0.7$ (Marino et al. 2011) and correspond to the MSa discovered by Bedin et al. (2004). Further information on MSa stars comes from the study based on multiwavelength 

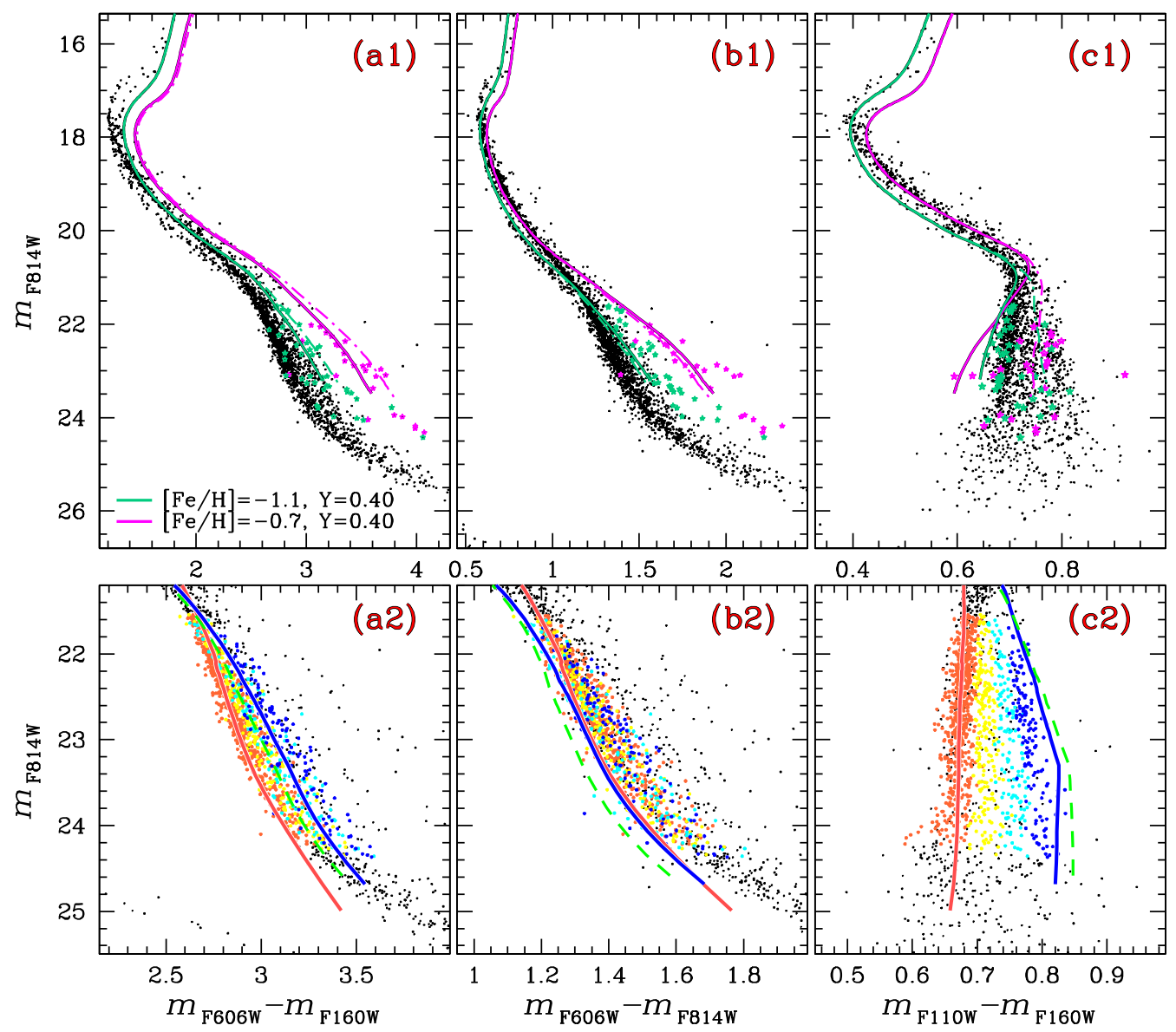

Figure 9. Upper Panels: reproduction of the CMDs of Fig. 5 with $S_{1}$ and $S_{2}$ stars coloured aqua and magenta, respectively. The aqua and magenta continuous lines are helium-rich isochrones with $[\mathrm{Fe} / \mathrm{H}]=-1.0$ and -0.7 , respectively. The corresponding dash-dotted isochrones account for the $\mathrm{C}, \mathrm{N}, \mathrm{O}$ abundance of $\mathrm{S}_{1}$ and $\mathrm{S}_{2}$ stars with extreme chemical composition. Lower Panels: zoom-in of the CMDs shown in the upper panels on the lower MS. Stars in the subpopulations A, B, C and D are coloured orange, yellow, cyan and blue, respectively. The isochrones introduced in Fig. 8 are overimposed on the CMDs. All the isochrones plotted in this figure have ages of $13.5 \mathrm{Gyr}$.

HST photometry by Bellini et al. (2010), who show that MSa stars are almost overimposed to the red MS in the $m_{F 814 W}$ versus $m_{F 606 W}-m_{F 814 W}$ plane. This fact demonstrates that MSa stars are highly helium enhanced with respect to the majority of stars in $\omega$ Centauri.

The magenta continuous isochrones overimposed to the CMDs of Fig. 9 have metallicity, $\alpha$-elements and helium abundances $([\alpha / \mathrm{Fe}]=0.4,[\mathrm{Fe} / \mathrm{H}]=-0.7$ and $Y=0.40)$ consistent with those inferred from the spectroscopic and photometric papers by Marino et al. (2011) and Bellini et al. (2010). The magenta dash-dotted isochrones have the same chemical composition as the continuous ones but are depleted in oxygen by $0.5 \mathrm{dex}$ and are derived as described in Section 5.1.

The aqua isochrones correspond to stellar populations with $[\mathrm{Fe} / \mathrm{H}]=-1.1$ and $[\alpha / \mathrm{Fe}]=0.4$ but different relative abundance of $\mathrm{C}, \mathrm{N}$ and $\mathrm{O}$. In this case, the dash-dotted isochrones are enhanced in $\mathrm{N}$ by 1.0 dex and depleted in carbon and oxygen by 0.6 and 0.4 dex with respect to the continuous isochrones. The adopted values of $\mathrm{C}, \mathrm{N}$ and $\mathrm{O}$ are consistent with those derived by Marino et al. (2012) from high-resolution spectroscopy.

The adopted isochrones qualitatively match the observed colours and magnitudes of stars in the upper panels of Fig. 9. In particular, the fact that $S_{1}$ and $S_{2}$ stars are distributed between the continuous and the dash-dotted isochrones in the $m_{F 814 W}$ versus $m_{F 110 W}-m_{F 160 W}$ CMD suggests that the most metal-rich populations of $\omega$ Centauri exhibit star-to-star oxygen variations.

The subpopulations of MS-I and MS-II are analysed in the lower panels of Fig. 9 where we use orange, yellow, cyan and blue colours to represent the stars of the four populations, A-D, identified in Section 4.1. We also overimpose to each CMD the isochrones introduced in Fig. 8. The four stellar subpopulations are well mixed in the $m_{F 814 W}$ versus $m_{F 606 W}-m_{F 814 W}$ CMD, but have different $m_{F 606 W}-m_{F 160 W}$ and $m_{F 110 W}-m_{F 160 W}$ colours.

As discussed in previous papers (e.g. Milone et al. 2012b, 2014; Dotter et al. 2015) and in Section 5.1, the $m_{F 110 W}-m_{F 160 W}$ colour is poorly sensitive to the stellar metallicity and the helium abundance, but is strongly affected by the oxygen abundance. The fact that the subpopulations A and B of MS-I exhibit, on average, different $m_{F 110 W}-m_{F 160 W}$ colours suggests that their stars mostly differ in their light-element abundance. Similar conclusions can be extended to the subpopulations $\mathrm{C}$ and D of the MS-II.

\section{SUMMARY AND CONCLUSIONS}

We have presented a study of multiple stellar populations in $\omega$ Centauri based on multi-epoch and deep images collected with the WFC/ACS and NIR/WFC 3 cameras of HST as part of the HST large programme on $\omega$ Centauri (GO-14118+14622, PI: L. R. Bedin). 
We have used the method based on effective PSF fitting (e.g. Anderson \& King 2000; Anderson et al. 2008) to derive highprecision stellar photometry and astrometry and determined stellar proper motions to separate cluster members from field stars.

The cluster CMD reveals multiple sequences over a range of at least eight magnitudes in the $F 814 W$ band. By using CMDs and pseudo-CMDs made with appropriate combinations of magnitudes, we have identified the two principal MSs (MS-I, and MS-II) of $\omega$ Centauri and for the first time we have followed them continuously, from the MS turn-off to the bottom of the MS. To investigate multiple populations among M-dwarfs, we have adapted to faint MS stars the method used by Milone et al. $(2015,2017)$ and we have derived pseudo-two-colour diagrams, or - by using the nomenclature of our previous papers - chromosome maps, by using NIR and optical colours. In this case, the pseudo-colour, $\Delta_{2}$, plotted on the $y$-axis of the chromosome map is derived from the $m_{F 110 W}-m_{F 160 W}$ colour and is mostly sensitive to stellar populations with different oxygen abundance. The quantity plotted on the $x$-axis, $\Delta_{1}$, is made by combining $F 606 W, F 814 W$ and $F 160 W$ magnitudes and it is also sensitive to the metallicity of the stellar populations.

We have discovered that below the MS knee, both MS-I and MSII host stellar subpopulations and we have identified at least four stellar groups A, B, C and D. Two additional stellar sequences $\left(\mathrm{S}_{1}\right.$ and $S_{2}$ ) are visible both in the chromosome map and on the red side of the most populated MSs in the $m_{F 814 W}$ versus $m_{F 606 W}-m_{F 160 W}$ CMD. They are consistent with $\alpha$-enhanced isochrones with $Y=$ 0.40 and $[\mathrm{Fe} / \mathrm{H}]=-1.1$ and -0.7 , respectively, and each of them is not consistent with a simple stellar population.

In CMDs made with optical $\left(m_{F 606 W}-m_{F 814 W}\right)$, NIR $\left(m_{F 160 W}\right.$ versus $\left.m_{F 110 W}-m_{F 160 W}\right)$ and mixed $\left(m_{F 606 W}-m_{F 160 W}\right)$ colours, the MS-I is redder than the MS-II in the upper part of the CMD and the two MSs merge together at the luminosity of the MS knee. In the NIR colour, the MS-I and the MS-II are well separated below the MS knee but their colour order is inverted, with the MS-I being redder than the MS-II. A similar MS pattern has been previously observed in the massive GC NGC 2808, where the two helium-rich MSs behave like the MS-II of $\omega$ Centauri, while the helium-normal MS would correspond to the MS-I (Milone et al. 2012b). The CMD of M-dwarfs in $\omega$ Centauri is more complex than that of previously studied GCs (e.g. Milone et al. 2012b, 2014; Richer et al. 2013; Correnti et al. 2016) and hosts at least six stellar subpopulations.

We have compared the observed CMDs with appropriate isochrones where we have accounted for the effect of metallicity, helium and $\mathrm{C}, \mathrm{N}, \mathrm{O}$ on the stellar colours. We confirm that the MS-I corresponds to a metal-poor stellar population $([\mathrm{Fe} / \mathrm{H}] \sim$ -1.7) with primordial helium abundance and high oxygen content ([O/Fe] 0.4). MS-II is well fitted both by a metal-poor and a metalrich isochrone with $[\mathrm{Fe} / \mathrm{H}]=-1.7$ and -1.4 . In order to reproduce the MS region above the knee, both isochrones should be strongly enhanced in helium, with the metal-poor and metal-rich isochrones having $Y=0.37$ and 0.40 , respectively. These results suggest that MS-II hosts helium-rich stars that span a wide range of metallicity.

\section{ACKNOWLEDGEMENTS}

This research is based on observations with the NASA/ESA Hubble Space Telescope, obtained at the Space Telescope Science Institute, which is operated by AURA, Inc., under NASA contract NAS 5-26555, under GO-14118 and GO-14662. We are grateful to the anonymous referee for comments and suggestions that have improved the quality of this manuscript. APM and AFM acknowledge support by the Australian Research Council through
Discovery Early Career Researcher Awards DE150101816 and DE160100851. JA, DA, AB, AJB, AD and JMR acknowledge the support for programme number GO-14118 and GO-14662 provided by NASA through a grant from the Space Telescope Science Institute.

\section{REFERENCES}

Anderson A. J., 1997, PhD thesis, Univ. California

Anderson J., King I. R., 2000, PASP, 112, 1360

Anderson J., King I. R., 2003, AJ, 126, 772

Anderson J. et al., 2008, AJ, 135, 2055

Bedin L. R., Piotto G., King I. R., Anderson J., 2003, AJ, 126, 247

Bedin L. R., Piotto G., Anderson J., Cassisi S., King I. R., Momany Y., Carraro G., 2004, ApJ, 605, L125

Bedin L. R., Cassisi S., Castelli F., Piotto G., Anderson J., Salaris M., Momany Y., Pietrinferni A., 2005, MNRAS, 357, 1038

Bedin L. R., Salaris M., Piotto G., Anderson J., King I. R., Cassisi S., 2009, ApJ, 697, 965

Bellini A., Bedin L. R., Piotto G., Milone A. P., Marino A. F., Villanova S., 2010, AJ, 140, 631

Bellini A., Anderson J., Salaris M., Cassisi S., Bedin L. R., Piotto G., Bergeron P., 2013, ApJ, 769, L32

Brown J. A., Wallerstein G., 1993, AJ, 106, 133

Cassisi S., Salaris M., Pietrinferni A., Piotto G., Milone A. P., Bedin L. R., Anderson J., 2008, ApJ, 672, L115

Castelli F., 2005, Mem. Soc. Astron. Ital. Suppl., 8, 25

Correnti M., Gennaro M., Kalirai J. S., Brown T. M., Calamida A., 2016, ApJ, 823, 18

D'Antona F., D'Ercole A., Marino A. F., Milone A. P., Ventura P., Vesperini E., 2011, ApJ, 736, 5

D’Antona F., Vesperini E., D’Ercole A., Ventura P., Milone A. P., Marino A. F., Tailo M., 2016, MNRAS, 458, 2122

Dotter A., Chaboyer B., Jevremović D., Kostov V., Baron E., Ferguson J. W., 2008, ApJS, 178, 89

Dotter A. et al., 2010, ApJ, 708, 698

Dotter A., Ferguson J. W., Conroy C., Milone A. P., Marino A. F., Yong D., 2015, MNRAS, 446, 1641

Gilliland R. L., 2004, Instrument Science Report ACS 2004-01, p. 18

Harris W. E., 1996, AJ, 112, 1487

Johnson C. I., Pilachowski C. A., 2010, ApJ, 722, 1373

Johnson C. I., Pilachowski C. A., Simmerer J., Schwenk D., 2008, ApJ, 681, $1505-1523$

Johnson C. I., Pilachowski C. A., Michael Rich R., Fulbright J. P., 2009, ApJ, 698, 2048

Karakas A. I., Marino A. F., Nataf D. M., 2014, ApJ, 784, 32

King I. R. et al., 2012, AJ, 144, 5

Kurucz R. L., 2005, Mem. Soc. Astron. Ital. Suppl., 8, 14

Lee Y.-W., Joo J.-M., Sohn Y.-J., Rey S.-C., Lee H.-C., Walker A. R., 1999, Nature, 402, 55

Lindegren L. et al., 2016, A\&A, 595, A4

Marino A. F., Piotto G., Gratton R., Milone A. P., Zoccali M., Bedin L. R., Villanova S., Bellini A., 2010, in Charbonnel C., Tosi M., Primas F., Chiappini C., eds, Proc. IAU Symp. 268, Light Elements in the Universe. Cambridge Univ. Press, Cambridge, p. 183

Marino A. F. et al., 2011, ApJ, 731, 64

Marino A. F. et al., 2012, ApJ, 746, 14

Milone A. P., 2015, MNRAS, 446, 1672

Milone A. P., Bedin L. R., Piotto G., Anderson J., 2009, A\&A, 497, 755

Milone A. P. et al., 2012a, A\&A, 540, A16

Milone A. P. et al., 2012b, ApJ, 754, L34

Milone A. P. et al., 2014, MNRAS, 439, 1588

Milone A. P. et al., 2015, MNRAS, 447, 927

Milone A. P. et al., 2017, MNRAS, 464, 3636

Norris J. E., 2004, ApJ, 612, L25

Norris J. E., Da Costa G. S., 1995, ApJ, 447, 680 
Pancino E., Ferraro F. R., Bellazzini M., Piotto G., Zoccali M., 2000, ApJ, 534, L83

Pancino E., Pasquini L., Hill V., Ferraro F. R., Bellazzini M., 2002, ApJ, 568, L101

Partridge H., Schwenke D. W., 1997, J. Chem. Phys., 106, 4618

Piotto G. et al., 2005, ApJ, 621, 777

Renzini A. et al., 2015, MNRAS, 454, 4197

Richer H. B., Heyl J., Anderson J., Kalirai J. S., Shara M. M., Dotter A., Fahlman G. G., Rich R. M., 2013, ApJ, 771, L15

Sbordone L., Bonifacio P., Castelli F., 2007, Convection Astrophys., 239, 71

Sbordone L., Salaris M., Weiss A., Cassisi S., 2011, A\&A, 534, A9

Schwenke D. W., 1998, Faraday Discuss., 109, 321

Silverman B. W., 1986, Monographs on Statistics and Applied Probability. Chapman and Hall, London

Sollima A., Pancino E., Ferraro F. R., Bellazzini M., Straniero O., Pasquini L., 2005, ApJ, 634, 332

Stanford L. M., Da Costa G. S., Norris J. E., 2010, ApJ, 714, 1001

Tailo M., Di Criscienzo M., D’Antona F., Caloi V., Ventura P., 2016, MNRAS, 457, 4525
Ventura P., Caloi V., D’Antona F., Ferguson J., Milone A., Piotto G. P., 2009, MNRAS, 399, 934

Villanova S. et al., 2007, ApJ, 663, 296

Villanova S., Geisler D., Gratton R. G., Cassisi S., 2014, ApJ, 791, 107

\section{SUPPORTING INFORMATION}

Supplementary data are available at MNRAS online.

Please note: Oxford University Press is not responsible for the content or functionality of any supporting materials supplied by the authors. Any queries (other than missing material) should be directed to the corresponding author for the article.

This paper has been typeset from a $\mathrm{T}_{\mathrm{E}} \mathrm{X} / \mathrm{L} \mathrm{T} \mathrm{E} \mathrm{X}$ file prepared by the author. 